\title{
Teat Dip/Spray Solution Dosage Form
}

National Cancer Institute

\section{Source}

National Cancer Institute. Teat Dip/Spray Solution Dosage Form. NCI Thesaurus. Code C149988.

Liquid preparation intended for use as a teat dip solution or as a teat spray solution. 